\title{
Erratum: Thermal Transport in the Kitaev Model [Phys. Rev. Lett. 119, 127204 (2017)]
}

Joji Nasu๑, Junki Yoshitake, and Yukitoshi Motome®

(A) (Received 15 February 2021; published 15 March 2021)

DOI: 10.1103/PhysRevLett.126.119901

There is an error in the expression of $\kappa_{\text {Kubo }}^{\mu \nu}(\omega)$ in the last paragraph on page 2 and Eq. (S23) in the Supplemental Material. The error is in the superscript $\mu$ and $\nu$ in the right-hand side. The correct expression is given as

$$
\kappa_{\mathrm{Kubo}}^{\mu \nu}(\omega)=\frac{1}{T V} \int_{0}^{\infty} d t e^{i(\omega+i \delta) t} \int_{0}^{\beta \hbar} d \lambda\left\langle J_{Q}^{\nu}(-i \lambda) J_{Q}^{\mu}(t)\right\rangle
$$

We set $\hbar=1$ in the last paragraph on page 2 . As all the numerical results were obtained from the above correct form, the error has no effect on our conclusions. 